QUADERNS DE FILOSOFIA VOL. VII NÚM. I (2020): 85-IO7

eISSN: 234I-3042 DOI: I O.7203/QFIA. 7.I.I 5787

JAVIER FABO LANUZA

Universidad Complutense de Madrid

\title{
Contribución a la traducción de la Ciencia de la lógica de Hegel al castellano (Observaciones a la edición de Félix Duque $)^{1}$
}

\author{
Contribution to Hegel's Science of Logic into spanish translation \\ (Remarks on Félix Duque edition)
}

Recibido: 13.9.2019 / Aceptado: 23.4.2020

Resumen: En este trabajo me propongo analizar, comentar y discutir algunas opciones traductoras adoptadas por F. Duque en su monumental edición de la Ciencia de la lógica. Concretamente, me centraré en la traducción de los términos alemanes: "Daseyn," "Unwesen," "Auslegung" y "ausgeführte [Zweck]," que cotejaré puntualmente con las principales traducciones extranjeras, así como con la castellana de $\mathrm{R}$. Mondolfo, no tanto para retomar su terminología, cuanto para valorar y calibrar mejor la de Duque, en la idea de que esta retrospectiva permite explorar otras posibilidades.

Abstract: In this paper I work up to analyze, comment and discuss some translation options adopted by F. Duque in his monumental edition of the Science of Logic. Specifically, I will focus on the translation of the german terms: "Daseyn," "Unwesen," "Auslegung" and "ausgeführte [Zweck]," which I will compare promptly with the main foreign translations, as well as with the one of R. Mondolfo, not so much to resume its terminology, as to assess and calibrate Duque's better, in the idea that this retrospective allows to explore other possibilities.

Palabras clave: Hegel, lógica, estar, antiesencia, exhibición, fin cumplido.

Keywords: Hegel, logic, being, antiessence, exhibition, fulfilled purpose.

${ }^{1}$ Esta nota desarrolla y amplía algunas ideas de mi tesis doctoral sobre la Ciencia de la Lógica de Hegel: Cf. Fabo Lanuza, J. Reflexión o diferencia. Un estudio sobre la cuestión "Hegel y el problema de la metafisica". 


\section{$\mathrm{E}$}

$S$ DE esPerar que en los próximos ańos el universo editorial hispanohablante vaya incorporando a su acervo los frutos del trabajo que el Hegel-Archiv de Buchum viene realizando durante décadas con la Gesammelte Werke [GW] de Hegel. El que la edición crítica de la Ciencia de la lógica de Hegel corra a cargo de un especialista en la materia como Félix Duque es una suerte que el investigador en lengua castellana no puede por menos que saludar con entusiasmo y agradecer. Adelantándose un año a la efeméride de su primer centenario, el primer volumen de este proyecto apareció en 2011 en la editorial Abada y recoge los dos libros de la "Lógica objetiva": la "Doctrina del ser" de 1812 y la "Doctrina de la esencia" de 1813. La traducción de la "Lógica subjetiva" de 1816 (junto con los suplementos de la edición crítica) aparecía cuatro años después en la misma editorial, completando así la monumental edición de la denominada "Gran lógica" o "Lógica Núremberg”. El proyecto original prevé también la publicación de un tercer volumen inédito hasta la fecha, que recogerá la segunda edición de la "Doctrina del ser" de 1932 y la "Notas doctrinales" a la entera Ciencia de la lógica (de las que el autor espera que puedan servir "de eso que antaño se llamaba Comentario per perpetuo o Vademecum"2). De cumplirse, el investigador contaría con una "traducción integral y científicamente suficiente"3 de esta obra que permitiría profundizar, no sólo en el texto de Hegel (lo que no es poco), sino también en su evolución a lo largo de los veinte años que median entre las dos ediciones de la "Doctrina del ser". La posibilidad de cotejar ambos textos permitirá comprender la evolución experimentada por la concepción hegeliana de la Lógica durante las etapas de Heidelberg y Berlín; asunto nada desdeñable, teniendo en cuenta que en esas etapas fueron redactadas las tres ediciones de la Enciclopedia de las ciencias filosóficas ( I 8 I 7, 27, 30), donde la Lógica experimenta modificaciones importantes que afectan tanto a su interna arquitectura como a su inserción en el conjunto del Sistema.

No es objeto de esta nota entrar a analizar estas modificaciones y su repercusión en la revisión de 1832. Confiamos a la erudición del traductor esta tarea que, a buen seguro, emprenderá con maestría en el tercer volumen de su proyecto. Entretanto, nos limitaremos a subrayar la importancia de que una edición como ésta vea la luz en el ámbito editorial hispanohablante, y de que lo haga de la mano de uno de los especialistas sobre idealismo alemán más reconocidos internacionalmente. La calidad de su trabajo se hace patente en la traducción de los dos volúmenes aparecidos a día de hoy, que actualizan y mejoran muchos aspectos de la que había sido la referencia principal hasta la fecha: la traducción de R. Mondolfo de 1956, que coteja las traducciones italianas de A. Moni y la francesa de V. Jankélévitch. La misma calidad se refleja en las amplias intro-

${ }^{2}$ Duque, F. (trad.), II, 9.

${ }^{3}$ Duque, F. (trad.), I, 13. 
ducciones que anteceden a las traducciones de los mencionados volúmenes. La primera se presenta como una contribución "a una lectura coherente actualizada y general de los grandes temas de la lógica hegeliana desde su acceso liminar en los prólogos de la obra respectivamente Núremberg y Berlín) y en el 'Concepto previo' de la Enciclopedia de 1827". La segunda reconstruye con todo lujo de detalles (como sólo un experto conocedor de la edición crítica podía hacer) el complejo proceso de gestación de la Ciencia de la lógica desde las primeras lecciones de Hegel sobre la materia en Jena, ofreciendo todo un "cuadro realista" capaz de contrarrestar el tópico del "sistema cerrado" tan recurrente en el hegelianismo hasta bien entrados los años setenta del pasado siglo. Especial mención merece también el aparato crítico de notas, glosarios e índices, que permite al lector orientarse en el complejo texto de Hegel, y que son el resultado de una investigación concienzuda, minuciosa y madura.

En esta nota, me propongo analizar, comentar y discutir algunas opciones traductoras adoptadas por F. Duque en esta edición de la Ciencia de la lógica, ciertamente arriesgadas en algunos casos, pero que son el fruto maduro de una larga trayectoria intelectual (tal y como testimonia su aparición en trabajos anteriores $\left.^{4}\right)$. Puntualmente, cotejaré estas opciones con las principales traducciones francesas, inglesas e italianas, así como con la traducción castellana de Mondolfo, no tanto para retomar su terminología, cuanto para valorar y calibrar mejor la de Duque, en la idea de que esta retrospectiva pudiera proporcionar, a la postre, claves interesantes para explorar otras posibilidades. Concretamente, consideraré las traducciones de los términos alemanes: "Daseyn", "Unwesen", "Auslegung" y "ausgeführte [Zweck?". La primera pertenece a la "Doctrina del ser”, la segunda y la tercera flanquean la "Doctrina de la esencia", y la tercera aparece en un lugar decisivo de la "Doctrina del concepto" (el final de la Teleología y el tránsito a la Idea). La siguiente tabla comparativa refleja la terminología que manejaremos:

\begin{tabular}{|c|c|c|c|}
\hline & R. Mondolfo & F. Duque & $\begin{array}{c}\text { Propuestas } \\
\text { Alternativas }\end{array}$ \\
\hline $\begin{array}{l}\text { Daseyn } \\
\text { bestimmtes Sein } \\
\text { bestimmtes Daseyn }\end{array}$ & $\begin{array}{l}\text { ser determinado, } \\
\text { existencia } \\
\text { id. } \\
\text { id. }\end{array}$ & $\begin{array}{l}\text { estar } \\
\text { ser determinado } \\
\text { estar determinado }\end{array}$ & $\begin{array}{l}\text { ser-determinado } \\
\text { ser determinado } \\
\text { determinado } \\
\text { ser-determinado } \\
\text { (/definido/limitado) }\end{array}$ \\
\hline Unwesen & no esencia & $\begin{array}{l}\text { contraesencia, } \\
\text { antiesencia }\end{array}$ & inesencia \\
\hline Auslegung & exposición & exhibición & $\begin{array}{l}\text { interpretación, } \\
\text { exposición } \\
\text { interpretativa }\end{array}$ \\
\hline
\end{tabular}

${ }^{4} C f$. Bibliografía: Otras referencias. 


\begin{tabular}{|l|l|l|l|}
\hline & \multicolumn{1}{|c|}{ R. Mondolfo } & \multicolumn{1}{|c|}{ F. Duque } & \multicolumn{1}{|c|}{$\begin{array}{c}\text { Propuestas } \\
\text { Alternativas }\end{array}$} \\
\hline $\begin{array}{l}\text { Ausgeführte } \\
\text { /erfüllte } \\
\text { /realisierte [Zweck] }\end{array}$ & $\begin{array}{l}\text { [fin] realizado } \\
\text { /realizado } \\
\text { /realizado }\end{array}$ & $\begin{array}{l}\text { [fin] cumplido } \\
\text { /cumplido } \\
\text { /realizado }\end{array}$ & $\begin{array}{l}\text { [fin] ejecutado } \\
\text { /cumplido } \\
\text { /realizado }\end{array}$ \\
Erfüllung & acabamiento & $\begin{array}{l}\text { llenado, } \\
\text { plenificación }\end{array}$ & cumplimiento \\
\hline
\end{tabular}

La diseminación de esta terminología a lo largo de la tópica lógica hace que este comentario no pueda ofrecer una interpretación demasiado elaborada de un aspecto concreto de la obra. Considero que cierto esfuerzo hermenéutico resulta, sin embargo, insoslayable, dada la estrecha relación que tiene en Hegel el lenguaje con la forma de pensar. Para atender a esta peculiaridad, me limitaré a esbozar una interpretación del contexto inmediato en el que aparecen los términos a comentar, lo que me llevará a moverme abruptamente de un extremo a otro de la obra de Hegel sin atender a su coherencia interna y unidad de sentido. El resultado será un análisis dirigido a mostrar la plausibilidad de las opciones traductoras propuestas, pero que en ningún caso pretende ser una lectura o interpretación de la lógica hegeliana.

\section{DASEYN}

Empezaré comentando la traducción de "Daseyn" como "estar", sobre la que Duque dispensa algunas aclaraciones en su estudio introductorio, y en el que reconoce abiertamente "el punto que más puede llamar la atención al lector de habla española". Esta opción corrige las traducciones de Mondolfo como "ser determinado" o "existencia", las cuales merecen, a mi juicio, diferente consideración. Como no podía ser de otra manera, suscribo las razones de Duque para rechazar la traducción de "Daseyn" como "existencia", reservando la expresión para traducir la palabra de origen latino "Existenz", que Hegel utiliza en la "Doctrina de la esencia". Mejor consideración me merece, en cambio, la traducción de "Daseyn" como "ser determinado", adoptada

${ }^{5}$ Duque, F. (trad.), I, 141.

${ }^{6} \mathrm{La}$ opción de la reciente traducción inglesa de G. di Giovanni de mantener el término "existence" (83) para "Daseyn" y traducir "Existenz" por "concrete existence" (420) me parece desafortunada. En primer lugar, porque el término "existence" introduce un matiz de exterioridad inexistente en el término "Daseyn". En segundo lugar, porque el adjetivo "concret" introduce un matiz de determinación y particularidad más apropiado al uso que Hegel hace del término "Daseyn" en la lógica de la cualidad que al que hace del término "Existenz". 
también por las primeras traducciones de A. Moni ${ }^{7}$ y W. H. Johnston ${ }^{8}$, pero que Duque rechaza en favor del término "estar" atendiendo a dos razones. La primera es que la opción de Mondolfo forzaría un pleonasmo en la traducción de la expresión hegeliana "bestimmtes Daseyn", que la coherencia terminológica obligaría a traducir como "ser determinado determinado" (o "determinado ser determinado"). La segunda tiene que ver con la incapacidad de esa opción traductora para preservar la diferencia con la expresión "bestimmtes Seyn”, cuya traducción más literal sería también "ser determinado".

Antes de entrar a valorar las posibles alternativas, conviene tener en cuenta que se trata de problemas puramente estilísticos, cuya dimensión conviene calibrar adecuadamente en el marco de un texto filosófico como el que nos ocupa, antes de administrar remedios que podrían ser peores que la enfermedad misma. A este respecto, tampoco resulta ocioso apuntar que la expresión “bestimmtes Daseyn" no aparece más de cinco veces en la Ciencia de la Lógica10, mientras que la expresión "bestimmtes Seyn" aparece un total de once veces, con un significado, por lo demás, muy próximo (si no estrictamente sinónimo) al de la expresión "Daseyn"11. Está claro que ello no nos dispensa de encontrar una traducción adecuada para estas expresiones, capaz de preservar en castellano los matices presentes en el texto original, pero nunca al precio de acabar sacrificando el contenido filosófico a la forma literaria; sobre todo en aquellos casos en los que ésta no tiene demasiada relación con la forma de pensar.

Atendiendo a este criterio, considero que el uso de la palabra "estar" no sólo proporciona un beneficio puramente estilístico, sino que tiene el inconveniente de borrar una resonancia terminológica presente en el texto de Hegel y que la traducción de Mondolfo permitía preservar en castellano. Me refiero a la resonancia de la palabra "Seyn" que reverbera en la palabra "Daseyn", y que deja entrever la conclusión del círculo dialéctico operado en este primer tramo de la "Doctrina del ser", donde la categoría del "Daseyn" irrumpe como una nueva "inmediatez" y como un "primer retorno" del Ser a sí mismo. La traducción de esta expresión como "ser determinado" permite preservar esta resonancia, vertiendo con ello al castellano el carácter especulativo de la lengua alemana tan elogiado por Hegel, y que debe primar sobre otros aspectos puramente

${ }^{7}$ Cf. Moni, A. (trad.), I, 107.

${ }^{8}$ Cf. Johnston, W. H. (trad.), I, 121.

${ }^{9}$ Duque, F. (trad.), I, 141.

${ }^{10}$ Cf. GW 11, 46, 50, 61, 244, 316.

${ }^{11}$ Ello se colige con facilidad del uso del término en los pasajes que cito a continuación: "Das Denken oder Vorstellen, dem nur ein bestimmtes Sein, das Dasein, vorschwebt [...]" (ibid., 48); "[...] in der erhellten Finsternis kann etwas unterschieden werden, weil erst das getrübte Licht und die erhellte Finsternis den Unterschied an ihnen selbst haben und damit bestimmtes Sein, Daseyn sind" (ibid., 50); cf. también: ibid., 46. 
estilísticos, pues es en ellos donde se pone verdaderamente a prueba la calidad de la traducción (y de nuestra propia lengua) para acoger y hacer transparente lo más peculiar de la escritura hegeliana.

$\mathrm{Si}$, a pesar de todo, se deseara pulir el estilo de la traducción, siempre cabría recurrir a la expresión "ser-determinado" (con guión) para traducir la palabra "Daseyn" (dando a entender con ello que la expresión castellana traduce una sola palabra), reservando la grafía sin guión para la expresión "bestimmtes Sein". Por lo que a los mencionados pleonasmos se refiere, también cabría evitarlos recurriendo a expresiones sinónimas de "ser determinado", como "ser definido" o "ser limitado". La primera opción parece acorde con el espíritu de la lengua alemana, teniendo en cuenta que "definición" se dice en alemán "Begriffbestimmung", pero también al universo semántico y conceptual de la "Doctrina del ser", donde la palabra "finitud" (Endlichkeit) aparece como una de las principales modulaciones categoriales del "Daseyn". Lo mismo cabe decir de la segunda opción traductora, que resulta igualmente aceptable atendiendo a la categoría del "límite" (Schranke), la cual encuentra acomodo en el mismo universo semántico para referirse al lado negativo de la cualidad.

Todavía cabría cuestionar, sin embargo, si precisamente esta cercanía semántica no haría también de esta solución un remedio peor que la propia enfermedad, toda vez que sugiere conexiones con categorías que, aunque están ciertamente muy relacionadas con la "Doctrina del ser", Hegel se esfuerza en separar. ¿¿Merece la pena diluir esta separación (que tanto cuesta detectar al lector en la gradación infinitesimal de la dialéctica del Ser) por simples cuestiones estilísticas, sobre todo cuando la ganancia es tan poca en este terreno? En lo que al estilo concierne, lo cierto es que expresiones como "determinado ser delimitado" o "determinado ser definido" no son (en cuanto al contenido) menos redundantes que las que Duque quiere evitar. Pero ¿acaso no lo es también la propia expresión alemana "bestimmtes Daseyn"?

Sea como fuere, lo primordial en la traducción debe ser preservar el significado de la palabra "Daseyn" en una constelación cercana al campo semántico de la determinación y de la cualidad ${ }^{12}$, sin recurrir a palabras susceptibles de

${ }^{12}$ No hay que olvidar que la palabra en cuestión aparece en un apartado que lleva por título "Bestimmtheit (Qualität)" (cf. ibid., 43). Tampoco resulta casual que sea precisamente en la categoría del Daseyn donde aparece la Anmerkung hegeliana a la célebre tesis de Spinoza "Omnis determinatio est negatio" (ibid., 76). Determinatio aparece ahí como una designación equivalente para Daseyn, y su traducción podría ser "determinación" (Bestimmung). El que Hegel no la utilice aquí se debe seguramente a que es una palabra demasiado general para designar una categoría concreta de la Lógica. Igualmente significativo es que hable de "Denkbestimmungen" en general y que esta terminología aparezca fundamentalmente en lugares como prólogos, introducciones y conclusiones. En el cuerpo propiamente dicho del texto, la palabra "Bestimmung" aparece como sustrato léxico de expresiones complejas como son: "Wechselbestimmung", "Zahlbestimmungen", "Gradbestimmung", etc. 
añadir connotaciones espurias a esta esfera, como en cierta manera haría a mi entender la palabra "estar", ciertamente cercana al campo semántico de la cualidad (pues, como el propio Duque señala ${ }^{13}$, tiene la significación de "estar 'marcado', determinado o definido de tal forma o de otra" ${ }^{14}$ ), pero que acarrea un fuerte matiz accidental y contingente, propio de los estados transitorios ("estoy enfadado", "estoy enfermo", etc.), cuando no también de localización espacial ("estoy en casa", "estoy en Madrid", etc.), que no acaba de encajar en este lugar de la Lógica ${ }^{15}$, tal y como el propio Hegel se esfuerza en precisar $^{16}$. Si bien esta connotación espacial parece adecuarse de entrada al significado del adverbio alemán "da", ésta no resulta pertinente en el marco de la "Cualidad", donde lo que importa es el carácter de la cualidad como tal. Parece claro que, en este contexto, la partícula " $d a$ " no puede referirse a ninguna localización espacial, sino al límite inherente a la determinación misma que es, necesariamente, determinada en cuanto tal, en cuanto a su ser, o lo que es lo mismo, "ser-determinado".

Esta traducción sigue siendo, por tanto, la más adecuada, toda vez que "ser" denota "esencia", mientras que "estar" denota más bien "estado". Mutatis mutandis, cabría decir que la palabra "ser" denota "permanencia", mientras que la de "estar" se aproxima más a la idea de "devenir". Tampoco ello resulta trivial en un marco como el de la Ciencia de la Lógica, donde "Werden" es el nombre de la categoría que antecede a la categoría del Daseyn. Conviene recordar que esta categoría irrumpe en el texto de Hegel como la asunción ${ }^{17}$ de la categoría del Werden, y que esa superación acontece como la pacificación del continuo paso del ser a la nada y de la nada al ser que tenía lugar en ella; ambos momentos se detienen ( $\mathrm{y}$, por así decir, reposan y permanecen ${ }^{18}$ ) en la categoría de Daseyn, donde reaparecen complementándose bajo la forma de lo positivo y lo negativo del "ser-determinado" o "determinación": como "determinación" propiamente dicha y "negación" ("toda determinación es negación"), o como "realidad" (realitas) y "límite".

${ }^{13}$ Cf. Duque, F. (trad.), I, 141.

${ }^{14} I d$.

${ }^{15}$ La misma observación resulta, a mi juicio, extensible a la opción traductora de P. Giuspoli, que traduce "Daseyn" como "esserci" (80).

${ }^{16}$ GW 11, 59: "Es ist nicht bloßes Sein, sondern Dasein; etymologisch genommen: Sein an einem gewissen Orte; aber die Raumvorstellung gehört nicht hierher. Dasein ist, nach seinem Werden, überhaupt Sein mit einem Nichtsein, so daß dies Nichtsein in einfache Einheit mit dem Sein aufgenommen ist. Das Nichtsein so in das Sein aufgenommen, daß das konkrete Ganze in der Form des Seins, der Unmittelbarkeit ist, macht die Bestimmtheit als solche aus."

${ }^{17}$ Mantengo la afortunada traducción de Duque para la palabra "Aufhebung".

${ }^{18}$ Ibid., 57: "Dies Resultat ist das Verschwundensein, aber nicht als Nichts; so wäre es nur ein Rückfall in die eine der schon aufgehobenen Bestimmungen, nicht Resultat des Nichts und des Seins. Es ist die zur ruhigen Einfachheit gewordene Einheit des Seins und Nichts. Die ruhige Einfachheit aber ist Sein, jedoch ebenso nicht mehr für sich, sondern als Bestimmung des Ganzen”. 


\section{UNWESEN}

Especial dificultad ofrece también la traducción del término "Unwesen", que aparece puntualmente en un lugar crucial de la "Lógica objetiva": el tránsito de la esfera del Ser a la esfera de la Esencia. Se trata de un tránsito complejo, que se prolonga a lo largo de las primeras categorías de esta esfera ${ }^{19}$. No puedo detenerme aquí en explicar el significado de esta progresión y su encaje en el conjunto de la "Lógica objetiva". Me limitaré a señalar que ésta transcurre a lo largo de una doble línea argumentativa que, en ocasiones, se superpone en el complejo texto de Hegel y que atiende al doble significado de la Aufhebung como "suprimir-cancelar" y "conservar-elevar".

La primera línea argumentativa expone la progresiva supresión del Ser como esfera independiente, mediante su asunción en la interioridad de la Esencia, en la que el Ser queda relegado al nivel de lo puramente nulo e inesencial; este "Ser asumido" es lo que Hegel llama "Unwesen"20. La segunda línea expone la conservación del Ser previamente asumido en el interior de la esencia (im Wesen), como "resto" (Rest) del Ser en esa esfera, y responde a lo que Hegel llama "apariencia" (Schein). El mismo doble proceso reaparece en el seno de esa misma categoría, que es caracterizada por Hegel como "resto" del Ser en la Esencia, pero también como "lo inmediato nulo en sí y por sí [das an und für sich nichtige Unmittelbare]"21.

Pues bien: esta "nulidad inmediata" se identifica con lo que en la categoría anterior Hegel había llamado "Unwesen", término que podría ser traducido sin mayor problema como "lo inesencial", de no ser por la conveniencia de reservar esa palabra para la categoría previa que Hegel denomina "das Unwesentliche". Esto es, en efecto, lo que hacen tanto Mondolfo como Duque, que se ven obligados a buscar una traducción alternativa para el término "Unwesen": mientras Mondolfo opta por la traducción (más literal) de "no esencia", Duque propone el neologismo de "antiesencia" 22 . Pretende matizar con ello el neologismo sinónimo de "contra-esencia", propuesto por él con anterioridad ${ }^{23}$, a fin

${ }^{19}$ A lo largo del tramo que va hasta la categoría de la "reflexión", y quizá incluso (según el criterio que se adopte) hasta las "determinaciones de reflexión". Cf. Fabo Lanuza, J., op. cit., 233-92.

${ }^{20}$ GW 11, 245-6 / (II), 17 (Mondolfo) / (I), 442-3 (Duque): "Das Wesen aber ist die absolute Negativität des Seins; es ist das Sein selbst, aber nicht nur als ein Anderes bestimmt, sondern das Sein, das sich sowohl als unmittelbares Sein wie auch als unmittelbare Negation, als Negation, die mit einem Anderssein behaftet ist, aufgehoben hat. Das Sein oder Dasein hat sich somit nicht als Anderes, denn das Wesen ist, erhalten, und das noch vom Wesen unterschiedene Unmittelbare ist nicht bloß ein unwesentliches Dasein, sondern das an und für sich nichtige Unmittelbare; es ist nur ein Unwesen, der Schein."

${ }^{21} \mathrm{Id}$.

${ }^{22}$ Cf. Duque, F. (trad.), I, nota 7.

${ }^{23}$ Cf. Duque, F., Historia de la filosofia moderna, 639. 
de evitar la superposición con el prefijo alemán "gegen-", cuya traducción más literal en castellano es justamente "contra-". El problema de ambas opciones es, sin embargo, su connotación excesivamente positiva y combativa, incapaz de reflejar el significado negativo del prefijo alemán "un-", y que es justamente lo que Hegel quiere evitar mediante el uso del término "Unwesen".

La mencionada connotación positiva de contraposición e independencia frente a la Esencia se ajustaría mejor a la primera categoría de la Esencia, a la que Hegel confiere una formulación antinómica: "Das wesentliche und das Unwesentliche" 24 . La presencia de esta antinomia se debe a que en ella todavía no se ha consumado el proceso de interiorización del Ser en la Esencia, con lo que éste permanece todavía contrapuesto a ella. La contraposición entre las esferas del Ser ("lo esencial") y la Esencia ("lo inesencial") perseverará hasta que no se consume el tránsito a la apariencia $(S c h e i n)^{25}$, cuyo umbral es precisamente el término "Unwesen": éste aparece al final de la dialéctica de lo esencial y lo inesencial precisamente para caracterizar -el punto de llegada del proceso de asunción del Ser en la nueva esfera de la Esencia, donde será preservado como lo negativo. La traducción literal de Mondolfo como "no-esencia" (que reproduce la traducción italiana de A. Moni ${ }^{26}$ y es secundada por la traducción inglesa de G. di Giovanni ${ }^{27}$ ) tiene la virtud de preservar este carácter negativo del Ser asumido y superado.

A falta de un término castellano más adecuado, cabría considerar también neologismos como "inesencia" o "desesencia", cuyos prefijos reflejan también el carácter negativo de la partícula alemana "un-". Concretamente, el término "inesencia" tiene la ventaja de ser el sustantivo correspondiente al adjetivo (sustantivado) de "(lo) inesencial", lo que permite verter al castellano el juego de palabras "unwesentlich"-"Unwesen" y apreciar así en nuestra lengua las sutiles modulaciones terminológicas empleadas por Hegel para expresar la continuidad del tránsito dialéctico a la apariencia. Sin embargo, Duque ha rechazado esta opción traductora, considerando que "traducir aquí 'inesencia' sería tan literal como ininteligible en español" 28 . Personalmente, considero que, como todo neologismo, la palabra resulta artificiosa, forzada y poco elegante en castellano, mas no ininteligible. Buen indicio de ello es que la longeva traducción inglesa W. H. Johnston utiliza el término inglés equivalente de "unessence"29 para traducir esta expresión.

${ }^{24} G W 11,245$.

${ }^{25}$ Ibid., 244: "Das Wesen aus dem Sein herkommend scheint demselben gegenüberzustehen; dies unmittelbare Sein ist zunächst das Unwesentliche. Allein es ist zweitens mehr als nur unwesentliches, es ist wesenloses Sein, es ist Schein."

${ }^{26}$ Moni, A. (trad.), II, 12.

${ }^{27}$ Giovanni, G. (trad.), 342.

${ }^{28}$ Cf. supr. nota 22.

${ }^{29}$ Johnston, W. H. (trad.), II, 21. 


\section{AUSLEGUNG}

El término "Auslegung" aparece al final de la "Lógica objetiva" como parte del sintagma "Auslegung des Absoluten". Como la mayoría de las traducciones extranjeras, Mondolfo lo traduce por "exposición" ${ }^{30}$, desdibujando con ello la diferencia con el concepto hegeliano de "Darstellung", para cuya traducción recurre también al mismo término. Para recuperar esta diferencia, Duque propone traducir "Auslegung" como "exhibición”. Las inevitables resonancias con la "exhibitio" kantiana y la omimoda determinatio escolásticoleibniziana que arrastra este término (y de las que el traductor es, por supuesto, plenamente consciente) $)^{31}$ resultan, sin embargo, a mi juicio, inapropiadas en el contexto de la Esencia, cuyo desdoblamiento estructural impide hacer una "exhibición" propiamente dicha de lo Absoluto, y donde de lo que se trata para Hegel es justamente de mostrar esta imposibilidad.

Atendiendo a este hecho, me parece más apropiada la traducción (más literal) de "interpretación", que el propio Duque reseña en una nota a pie, si bien sólo para rechazarla a causa de su connotación subjetiva ${ }^{32}$. Considero, sin embargo, que esta connotación subjetiva (propia de la subjetividad abstracta todavía contrapuesta a la objetividad) no resulta del todo inadecuada en una esfera como la de la Esencia, en la que lo lógico todavía no ha conseguido abandonar la dualidad de lo objetivo y lo subjetivo, siendo ésta la razón de que no pueda alcanzar la mencionada exhibición.

Lo que en su lugar viene a primer plano en las últimas páginas de la Esencia es el contramovimiento de la "realidad efectiva" (Wirklichkeit), en el que se superponen dos exposiciones equivalentes o indiferentes (gleichgültig) de lo Absoluto, o lo que es lo mismo: dos "interpretaciones" de lo Absoluto cuya referencia unitaria permanece todavía oculta en el fondo fotófobo (lichtscheues) de la Esencia. Nada más alejado, por tanto, de la singularidad del Concepto, en la que convergen y se unifican los momentos de la particularidad y la universalidad, y en la que cabe empezar a reconocer la verdadera exhibición de lo Absoluto que alcanzará pleno cumplimiento en la Idea absoluta.

El desdoblamiento de la Auslegung debe ser considerado como el punto de llegada más acabado del desdoblamiento de la Esencia. Éste se configura en las "determinaciones de reflexión" (cada una de las cuales contiene el todo

${ }^{30}$ Mondolfo, R. (trad.), I, 189.

${ }^{31}$ Duque, F., La especulación de la indigencia, 26.

32 Duque, F. (trad.), I, nota 231: “Auslegung (desde una posición teórica subjetiva, significa también 'exégesis', 'interpretación'. Representativamente hablando, aquí se pide que lo absoluto haga la exégesis de sí mismo, que ponga en claro lo que él es)." 
de la reflexión) y resulta visible en la división general de la Esencia: en la dualidad de la Esencia (la "reflexión en sí misma") y de la Existencia (la "reflexión fuera de sí"), cuya función no es otra que la de asumir en el interior de esta esfera la relación entre las dos esferas de la "Lógica objetiva, donde reaparecen bajo la forma de un doble proceso: como interiorización del Ser en la Esencia y como exteriorización de la Esencia en la Existencia. Ambos procesos deben ser considerados como aspectos complementarios de un mismo contenido absoluto que pugna por emerger en la Esencia, pero que todavía no logra hacerlo plenamente en ella, donde permanece todavía oculto como el secreto gozne de ese contra-movimiento.

Para Hegel no basta simplemente con pensar el contenido absoluto, todavía es preciso pensarlo absolutamente, vale decir: de forma absoluta. Una exigencia como ésta es la que inspira la autocomprensión de su idealismo absoluto como superación de los idealismos subjetivo y objetivo de Fichte y Schelling. En tanto que idealistas, ambas filosofías alcanzan a pensar el contenido absoluto, mas no a pensarlo de forma absoluta (vale decir: especulativamente). Como no podía ser de otra manera, ello requiere de la superación de ese régimen de dualidades característico de la "Lógica objetiva". Si la dialéctica de la Esencia permite pensar la copertenencia de sus momentos contrapuestos (mostrando cómo cada uno de ellos contiene en sí mismo la totalidad), ello es sólo desde el punto de vista del contenido. Por lo que a la forma respecta, la estructura espejada de la Esencia permanece en la indiferencia (Gleichgültigkeit) y el desdoblamiento (Verdoppelung) característico de esta esfera.

Ello vale incluso para la realidad efectiva, donde Hegel parece pensar no sólo la reciprocidad entre los momentos contrapuestos de la Esencia, sino también la unidad de los diferentes modos de pensar esa unidad; no es de extrańar, por tanto, que la última sección de la Esencia se corresponda con lo que tradicionalmente era denominado "lógica modal", y que uno de sus interlocutores privilegiados sea precisamente B. Spinoza: también para él se trataba, en efecto, de pensar la unidad de los modos en la unidad de una misma sustancia absoluta. Al igual que la sustancia spinoziana, lo Absoluto es pensado aquí como el punto de inherencia de los modos, como aquello que los hace ser modos de lo mismo. La realidad efectiva introduce con ello una identidad, que supera por primera vez el estrato de la igualdad y de la indiferencia propias de la Esencia, y que debe ser considerada como una primera aparición de lo Absoluto en el orden categorial. Esta es la razón de que el capítulo primero de la realidad efectiva lleve por título "Das Absolute", y que dicho capítulo plantee el problema de la "Auslegung des Absoluten": 
En la medida en que ese negar y este poner pertenecen $a$ la reflexión externa, ello es entonces una dialéctica formal asistemática, que a poca costa echa mano aquí y allá de determinaciones varias, y de igual manera hace ver a poca costa, de un lado, su finitud y mera relatividad, y de otro lado, al ser avistado por ella lo absoluto como la totalidad, enuncia también de él la inhesión de todas las determinaciones, sin poder elevar estas posiciones y esas negaciones a una unidad de verdad. Pero lo que lo absoluto sea es algo que debe venir expuesto [dargestellt werden]; sólo que este exponer [Darstellen] no puede ser un determinar ni reflexión externa, por cuyo medio vinieran a darse determinaciones del mismo, sino una exhibición [Auslegung], y además la propia exhibición [Auslegung] de lo absoluto, sólo un acto de mostrar [Zeigen] aquello que él es ${ }^{33}$.

Que la palabra "Auslegung" sea contrapuesta en este pasaje a la "reflexión extrínseca" da una idea de lo alejado que está el término de una "ex-posición", y de lo inadecuada que resulta, por tanto, la traducción del Mondolfo. A diferencia de la exposición (Darstellung), en la que el contenido es puesto fuera (ex-puesto), la Auslegung parece apuntar hacia una suerte de manifestación de la cosa misma; si bien todavía no a la verdadera automanifiestación de la cosa misma a partir de sí misma. Una automanifestación como ésta es algo que no puede tener lugar plenamente en el marco de la Esencia, sino solamente en el del Concepto, donde la exposición adopta la forma propiamente especulativa del (auto-)despliegue inmanente (Entwicklung). Esta limitación de la Esencia es lo que Hegel da a pensar mediante el uso de la palabra "Zeigen" ("indicar", "mostrar"): lo Absoluto no llega a manifestarse él mismo en las dualidades de la Esencia, pero puede ser señalado como algo que subyace a ellas y sin lo cual no podrían ser consideradas manifestaciones de lo mismo. De ahí que la palabra escogida por Hegel para designar este modo de aparición de lo Absoluto no sea "Entwicklung", sino precisamente "Auslegung", que propongo traducir por "interpretación" ${ }^{34}$, o también por "exposición interpretativa”, teniendo en cuenta la generalidad con la que Hegel utiliza el término "Darstellung" 35 . Las razones que subyacen a esta opción traductora nos obligan a extendernos algo más en estas explicaciones sobre el tramo final de la Esencia y el tránsito al Concepto.

${ }^{33} G W 11,370$ / 189 (Mondolfo) / I, 592 (Duque).

${ }^{34}$ Secundamos en este punto la traducción francesa de V. Jankélévitch, que traduce el término "Auslegung" por "interprétation" (II, 83).

${ }^{35}$ La traducción más literal de este término es la palabra latina "exposición". Esta palabra introduce una connotación de exterioridad que se ajusta muy bien al carácter de la Esencia, más no al del Concepto. El problema es que Hegel utiliza la palabra "Darstellung" también en esa esfera, lo que da una idea de la generalidad con la que utiliza el término. Nada impide, por tanto, modular el significado general de la palabra mediante el uso de adjetivos, como proponemos en el caso de la expresión "exposición interpretativa". 
Empezaremos señalando que la palabra "interpretación" alude, en general, a un determinado punto de vista sobre algo y, por tanto a una manera limitada de ver o entender su significado; toda interpretación se efectúa desde un determinado ángulo que no agota el significado de lo interpretado y que admite, por tanto, otras interpretaciones complementarias de dicho significado. Lo interesante de la palabra en cuestión es que (al igual que la Auslegung de la Esencia) preserva intacta la referencia unitaria del contenido de referencia (y, con ello, su carácter absoluto), pero sólo a condición de mantenerlo oculto, como algo que no emerge a la luz en la pluralidad de las interpretaciones; todas las interpretaciones revelan aspectos de lo mismo, pese a que ese "lo mismo" no emerge como tal en ninguna de ellas. Esto es justamente lo que ocurre con la sustancia de Spinoza, que permanece oculta bajo la superficie de sus modos, y a la que no por casualidad Hegel dedica la Anmerkung correspondiente al apartado de la "Realidad efectiva" donde desarrolla la cuestión de la Auslegung $g^{36}$.

$\mathrm{Al}$ igual que la sustancia, lo Absoluto no puede permanecer escindido en su manifestación (debe ser sí mismo incluso en lo otro de sí mismo). De ahí que dicha manifestación sólo pueda tener lugar en un singular, y que la palabra "exhibición" parezca de entrada adecuada a lo Absoluto hegeliano. El problema es que lo singular permanece todavía oculto tras las dualidades de la Esencia, incluso en el capítulo final de la misma (la "Realidad efectiva"), donde de lo que se trata es, ciertamente, de pensar la unidad del Ser y la Esencia, pero sólo en la forma limitada de esa esfera, irremediablemente escindida por la doble interpretación que admite esa relación, y a la que nos hemos referido como interiorización del Ser en el abismo de la Esencia y exteriorización de la Esencia en la superficie la Existencia.

El resultado es una doble manifestación en la que lo Absoluto aparece escindido en la dualidad de sus modos (o interpretaciones), y cuya unidad (singularidad) sólo puede ser mostrada o indicada como su referente oculto: como algo en relación a lo cual ambas son, ciertamente, interpretaciones de lo mismo (modos de la misma Sustancia), mas no como un referente unitario que se manifestara y, por así decir, se "exhibiera" como tal a través de esos modos, lo que requeriría la elevación de la sustancia al sujeto, y sólo puede tener lugar en la "Lógica subjetiva". Lo decisivo en el desenlace final de la "Lógica objetiva" reside en dilucidar si la opacidad de este referente común nos sitúa ante una suerte de exposición unitaria (aunque oscura) de la Esencia que refrendaría, a la postre, la traducción de "Auslegung" por "exposición", o resulta más bien incompatible con toda idea de unidad, abocándonos a la dualidad de las "interpretaciones" y, de este modo, a una manifestación que en modo alguno puede ser Absoluta. Lo primero parece

${ }^{36} G W 11,376$ y ss. 
colegirse del hecho de que Hegel presente la Realidad efectiva como la "totalidad de lo interior y lo exterior" y la "identidad de lo interno y lo externo":

Pero lo absoluto mismo es la identidad absoluta; eso es su determinación, mientras que toda la multiforme variedad del mundo que es en sí y del mundo fenoménico, o de la totalidad interior y exterior, está asumida [aufgehoben] dentro de él. Dentro de él mismo no hay ningún devenir, pues él no es el ser, ni tampoco el determinar reflexionante de sí; pues él no es la esencia, sólo en sí determinante de sí, ni tampoco un acto de externalizarse [ein Sich-Äußern]; pues él se da como la identidad de lo interno y lo externo ${ }^{37}$.

Pero esta "totalidad de lo interior y lo exterior" no parece poder erigirse en verdadera identidad (o identidad absoluta) mientras ambos momentos se mantengan en ella como dos maneras de pensar la totalidad de la Esencia. En tanto que momentos de la realidad efectiva, Ser y Esencia aparecen, por lo pronto, como dos maneras complementarias de suturar el desgarramiento de su propia esfera. Su complementariedad supera la contraposición de la apariencia y la esencia que regía las primeras categorías de la Esencia, pero sólo al precio de caer en indiferencia (Gleichgültigkeit) característica de esta esfera, y cuya máxima expresión es el elenco de relaciones simétricas (o "relaciones recíprocas") que Hegel estudia en la "Realidad efectiva". Según este esquema, la misma relación (de exteriorización) que lleva a la exterioridad del Ser a plegarse sobre sí misma para generar la interioridad de la Esencia, lleva también a esta interioridad a vaciarse y desfondarse en la exterioridad de la Existencia. Ambas maneras de pensar la unidad de la Esencia (como relación de lo interior y lo exterior) pertenecen a la Esencia y expresan la verdad de la Esencia como desdoblamiento. Pero el desdoblamiento que desde el punto de vista de la Esencia se presenta como lo más elevado, aparece desde el punto de vista superior del Concepto como una "doble apariencia" (Doppelschein) y, por tanto, como una manera limitada y unilateral de pensar la unidad absoluta:

Esa determinidad es, en efecto, en cuanto que está en el concepto, la reflexión total, la doble apariencia [Doppelschein]: de una parte, apariencia hacia afuera, la reflexión en otro; de otra, apariencia hacia dentro, la reflexión en sî́

${ }^{37}$ Ibid., 371 / II, 190 (Mondolfo) / I, 594 (Duque): "Aber das Absolute selbst ist die absolute Identität; dies ist seine Bestimmung, indem alle Mannigfaltigkeit der an sich seienden und der erscheinenden 'Welt oder der innerlichen und äußerlichen Totalität in ihm aufgehoben ist. In ihm selbst ist kein Werden, denn es ist nicht das Sein, noch ist es das sich reflektierende Bestimmen; denn es ist nicht das sich nur in sich bestimmende Wesen; es ist auch nicht ein Sich-Äußern, denn es ist als die Identität des Inneren und Äußeren."

${ }^{38}$ Ibid., 35 / II, 282 (Mondolfo) / II, 155 (Duque). 
El fragmento de la cita se refiere a la unidad de lo universal y lo particular en el Concepto, pero sólo en la medida en que éste no es pensado todavía bajo la unidad (la "exhibición" propiamente dicha) de lo singular. La misma doble apariencia caracteriza por tanto a la unidad de lo externo y lo interno propia de la "realidad efectiva", que todavía no ha alcanzado dicha unidad. Ésta permanecerá sumida en la noche de la sustancia mientras sea pensada en el marco de la lógica modal. No es de extrańar que la crítica de la sustancia spinoziana presida el tránsito al Concepto, y que la crítica de la "cosa en sî" kantiana aparezca también en las mismas páginas. Ambos conceptos se refieren a un Absoluto en negativo, que se limita a ser la referencia común de los modos (Hegel llega a hablar de la "negative Auslegung des Absoluten" ${ }^{\prime \prime}$ ). Por cuanto que permanece supeditada a sus modos, la unidad de la "realidad efectiva" no es más que la ciega necesidad de la Esencia que aborrece la luz (lichtschenes Wesen) y que los vincula sin saber cómo ni por qué.

Se colige de ahí que lo que propiamente aparece, se revela y accede a la plena manifestación no es la Esencia (como repiten las lecturas postmetafísicas de Hegel en su intento de minimizar la relevancia de la "Lógica subjetiva", en la que creen reconocer un desenlace dogmático y mistificador de la lógica hegeliana), sino el Concepto. Lo que aparece en la Erscheinung de la Esencia no es lo Absoluto, sino únicamente una modalidad de la Esencia: la Esencia que es sólo "reflexión en sí misma", y que todavía debe emerger a la superficie sin espesor de la existencia. Hegel declara que la Esencia debe (muss) aparecer, pero mientras que su Auslegung se limite a mostrar sus modos de aparición será preciso concluir que, aunque debe, la Esencia no puede aparecer; no al menos completamente y sin resto (vale decir: absolutamente). Así como el Ser no puede ser asimilado sin resto en la interioridad de la Esencia, así tampoco la Esencia puede emerger completamente a la superficie de la Existencia, no pudiendo evitar derivar en el contramovimiento o el contragolpe (Gegenstoss) de la "realidad efectiva". La emergencia de la Esencia deja siempre un trasfondo no asimilado, un excedente inexpresado que permanece, como un punto ciego, instalado en el oscuro gozne de su doble hélice reflexiva. Ese punto ciego y fotófobo es lo que se interpone en la "exhibición absoluta" de la Esencia, abocándola al desdoblamiento y a la doble interpretación (Auslegung) de sus modos.

\section{AusGeFÜHRTE/ERFÜLLTE/REALISIERTE [ZWECK]}

El adjetivo "asgeführte" es utilizado por Hegel en el apartado de la "Lógica subjetiva" titulado "Teleología", como adjetivo del sustantivo "Zweck", para

${ }^{39}$ Ibid., 371 / II, 191 (Mondolfo) / I, 594 (Duque). 
expresar lo que Hegel denomina "la objetividad del fin mismo"40, "su retorno objetivo a sî"41. La expresión "[der] ausgeführte Zweck" (que da título al tercer apartado de la Teleología) es traducida por Mondolfo como "fin realizado", mientras que Duque la traduce como "fin cumplido". El principal problema de ambas traducciones es que ignoran el matiz introducido por Hegel mediante el uso del adjetivo "erfüllte" 42 en el mismo apartado, así como del adjetivo "realisierte" en el tránsito a la Idea inmediata.

La opción traductora de Duque resulta especialmente problemática por lo que al primer matiz respecta, ya que asigna a la expresión "ausgeführte Zweck" la palabra más adecuada para traducir la expresión "erfüllte Zweck". Menos problemática parece resultar a este respecto, en cambio, la traducción de Mondolfo, que podría mantenerse para la palabra "ausgeführte", pero sólo a condición de adoptar la opción de Duque para traducir "erfüllte". Ahora bien: tampoco esta opción estaría exenta de inconvenientes, si tenemos en cuenta que la expresión "fin realizado" es la más adecuada para traducir la expresión "realisierte Zweck", que Hegel utilizará un poco más adelante, en la esfera de la Idea.

Para salvar estos matices terminológicos, propongo la traducción más literal de "fin ejecutado" ("ausführen" significa, ciertamente, "llevar a cabo", "acometer", "ejecutar") para la primera expresión, reservando para la segunda ("erfüllte Zweck") la traducción de "fin cumplido" "33. Pretendemos preservar así un importante matiz presente en el tramo final de la Teleología, pero que ha pasado generalmente desapercibido para traductores e intérpretes: la diferencia entre el fin que se ejecuta con el medio (y que preserva por tanto el medio exterior como momento independiente del Concepto) y la del fin que se cumple en el medio ( $\sin$ la concurrencia de un medio exterior).

No es en modo alguno casual que el término "erfüllen" aparezca por primera vez en un lugar donde Hegel habla del desgaste y la supresión del

${ }^{40} G W 12,165$.

${ }^{41} I d$.

${ }^{42}$ Ibid. 171 / II, 469 (Mondolfo) / II, 309 (Duque): "Diese Reflexion aber, daß der Zweck in dem Mittel erreicht und im erfüllten Zwecke das Mittel und die Vermittlung erhalten ist, ist das letzte Resultat der äußerlichen Zweckbeziehung, worin sie selbst sich aufgehoben und das sie als ihre Wahrheit dargestellt hat."

${ }^{43}$ Nos aproximamos así a las traducciones inglesas de Johnston y di Giovanni, que demuestran ser más sensibles a esta distinción, al traducir la expresión "erfüllte Zweck" por "fulfilled end" (II, 394) o "fulfilled purpose" (669). El problema de estas traducciones es que no reflejan la diferencia entre "ausgeführte" y "realisierte Zweck", al traducir indiferentemente ambas expresiones como "realiced end" o "realiced purpose". Lo mismo cabe decir de la traducción de Labarrière y Jaczyk, que traduce la primera expresión como "fin remplie" (III, 402), pero mantiene la traducción del "fin realisée" para las dos últimas. 
medio de la relación teleológica ${ }^{44}$, pues lo que en esta supresión se anticipa de alguna manera es el cumplimiento del fin, que requiere la desaparición del medio como algo externo e independiente. Lo mismo cabe decir del objeto, mientras se limite a ser algo contrapuesto al sujeto y no desempeñe otra función que la de mero medio para la satisfacción del fin exterior a él ${ }^{45}$. En tanto que retorno del fin a sí mismo, el cumplimiento del fin requiere de la superación de toda exterioridad (tanto del medio, como del objeto), lo cual sólo puede tener lugar mediante su propia realización u objetivación. Sólo a esta realización del fin en el objeto cabe denominar, conforme a la terminología hegeliana, "fin cumplido" (erfüllte ${ }^{46}$; mientras el fin precise de medios externos (o tome al objeto como un mero medio externo), el fin será, ciertamente, "ejecutado" (ausgeführte) (a través de esos medios), mas no propiamente "cumplido" 47.

${ }^{44}$ GW 12, 168-9 / II, 465 (Mondolfo) / II, 306 (Duque): "Was zur Ausführung eines Zwecks gebraucht und wesentlich als Mittel genommen werden soll, ist Mittel, nach seiner Bestimmung aufgerieben zu werden. Aber auch das Objekt, das den ausgeführten Zweck enthalten und sich als dessen Objektivität darstellen soll, ist vergänglich; es erfüllt seinen Zweck ebenfalls nicht durch ein ruhiges, sich selbst erhaltendes Dasein, sondern nur insofern es aufgerieben wird, denn nur insofern entspricht es der Einheit des Begriffs, indem sich seine Äußerlichkeit, d. i. seine Objektivität in derselben aufhebt. Ein Haus, eine Uhr können als die Zwecke erscheinen gegen die zu ihrer Hervorbringung gebrauchten Werkzeuge; aber die Steine, Balken, oder Räder, Achsen usf., welche die Wirklichkeit des Zweckes ausmachen, erfüllen ihn nur durch den Druck, den sie erleiden, durch die chemischen Prozesse, denen sie mit Luft, Licht, Wasser preisgegeben sind und die sie dem Menschen abnehmen durch ihre Reibung usf. Sie erfüllen also ihre Bestimmung nur durch ihren Gebrauch und Abnutzung und entsprechen nur durch ihre Negation dem, was sie sein sollen. Sie sind nicht positiv mit dem Zwecke vereinigt, weil sie die Selbstbestimmung nur äußerlich an ihnen haben, und sind nur relative Zwecke oder wesentlich auch nur Mittel."

${ }^{45}$ Ibid., 165: "Insofern die Tätigkeit wieder bloß darin bestünde, die unmittelbare Objektivität zu bestimmen, so würde das Produkt wieder nur ein Mittel sein und so fort ins Unendliche; es käme nur ein zweckmäßiges Mittel heraus, aber nicht die Objektivität des Zweckes selbst. Der in seinem Mittel tätige Zweck muß daher nicht als ein aber nicht die Äußerliches das unmittelbare Objekt bestimmen, somit dieses durch sich selbst zur Einheit des Begriffes zusammengehen; oder jene äußerliche Tätigkeit des Zwecks durch sein Mittel muß sich als Vermittlung bestimmen und selbst aufheben."

${ }^{46} \mathrm{El}$ adjetivo aparece aquí utilizado en el mismo sentido que en la expresión "erfüllte Kopula", utilizada por Hegel para referirse a la unificación de los extremos del sujeto y el predicado, contrapuesto en la forma del juicio, pero unificados gracias al silogismo en la unidad del concepto (ibid., 88 / II, 355 (Mondolfo) / II, 215 (Duque)); y también que en la expresión "erfülltes Sein", que el filósofo utiliza para referirse a la "totalidad intensiva" del método (ibid., 252 / II, 582 (Mondolfo) / II, 403 (Duque)).

${ }^{47}$ Especialmente significativo en este sentido resulta el hecho de que el verbo alemán "erfüllen" no se utilice tanto en relación con fines (que requieren de medios para su cumplimiento), cuanto para deseos, cuya relación el objeto es más inmediata; se dice, en efecto, "ein Wunsch erfüllen" y no "ein Wunsch ausführen". Por la misma razón, el Genio de Aladino, que no necesita de medios para cumplir los deseos de su amo, concede tres "deseos" y no tres "fines". 
Este cumplimiento es algo que no puede tener lugar en la relación medios-fines de la teología, sino sólo en la mediación absoluta de la Idea. No por casualidad, Hegel titula el apartado que nos ocupa "Der ausgeführte Zweck" (y no "Der erfüllte Zweck"). La aparición del término "erfüllte" al final de este apartado no hace sino propiciar el tránsito a la Idea inmediata. Tampoco es casual que precisamente ahí aparezca la palabra "Erfüllung", justamente para designar la superación del Concepto (de la escisión entre el Concepto subjetivo y la Objetividad) en la unidad absoluta de la Idea ${ }^{48}$. Mondolfo traduce el término por "acabamiento", mientras que Duque lo hace (más literalmente) por "llenado" o "plenificación”. Mantiene con ello la coherencia terminológica con la expresión "erfüllte Kopula" utilizada por Hegel en el tránsito al silogismo (cosa que Mondolfo no hace), pero sólo al precio de perderla con el término (textual y semánticamente más próximo) de "erfüllte Zweck". La traducción deja escapar con ello una interesante resonancia presente en el texto de Hegel, donde la transparencia misma del lenguaje dejar entrever el motivo central que conduce de la Teleología a la Idea inmediata.

Si el Concepto aspira a la unidad de lo subjetivo y lo objetivo en el elemento de la subjetividad abstracta operada sin la abstracta cópula del juicio, la Idea aspira a la unidad de la Subjetividad y la Objetividad misma, realizada sin la gestión del medio exterior de la Teleología. Los infinitesimales matices que el lenguaje especulativo de Hegel introduce en el proceso dialéctico que va desde la abstracta y oscura cópula del juicio hasta la determinada y transparente mediación (Vermittlung) de la Idea, pasando por el determinado (pero abstracto) término medio (Mitte) y el determinado (pero externo) medio teológico (Mitel), son de todo menos triviales en un contexto como el de la "Lógica subjetiva", que debe ser considerada como un "tratado del medio/método". Un estudio detallado ${ }^{49}$ de esta parte de la Lógica revelaría cómo el centro invisible del Concepto inmediato va cobrando presencia y densidad a lo largo del Concepto subjetivo y de la Objetividad, para disolverse finalmente en la mediación absoluta de la Idea, retornando así a la inmediatez del comienzo ${ }^{50}$.

${ }^{48}$ GW 12, 382 / II, 484 (Mondolfo) / II, 322 (Duque): "Die Bestimmungen des Gegensatzes sind die allgemeinen Bestimmungen des Begriffs, denn es ist der Begriff, dem die Entzweiung zukommt; aber die Erfüllung derselben ist die Idee. Das eine ist die Einheit des Begriffs und der Realität, welche die Idee ist, als die unmittelbare, die sich früher als die Objektivität gezeigt hat."

${ }^{49}$ Cf. Fabo Lanuza, J., op. cit., 375-406.

${ }^{50} G W 12$, 252: "So ist denn auch die Logik in der absoluten Idee zu dieser einfachen Einheit zurückgegangen, welche ihr Anfang ist; die reine Unmittelbarkeit des Seins, in dem zuerst alle Bestimmung als ausgelöscht oder durch die Abstraktion weggelassen erscheint, ist die durch die Vermittlung, nämlich die Aufhebung der Vermittlung zu ihrer entsprechenden Gleichheit mit sich gekommene Idee. Die Methode ist der reine Begriff, der sich nur zu sich selbst verhält; sie ist daher die einfache Beziehung auf sich, welche Sein ist. Aber es ist nun auch erfülltes Sein, der sich begreifende Begriff, das Sein als die konkrete, ebenso schlechthin intensive Totalität." 
Dada la escasa literatura filosófica sobre el tema (y el escaso acuerdo reinante en ella), considero oportuno concretar algo más este punto. Ya hemos apuntado en qué medida la Idea constituye el verdadero "erfüllte Zweck" anunciado en la Teleología, pero que ella no podía llegar a alcanzar so pena de superarse a sí misma; la principal razón es que lo que ella concebía como objeto exterior al fin aparece en la Idea como la realización del propio fin, y que lo que ella concebía como medio independiente tanto del objeto como del fin subjetivo, aparece en ella como mediación, como un momento susceptible de ser consumido en el retorno del fin hacia sí mismo ${ }^{51}$. Especialmente ilustrativo en este punto resulta el funcionamiento de la Vida, donde las células, los órganos y los individuos intervienen (con su funcionamiento, su desgaste y, finalmente, su muerte) como medios para la perpetuación del género, si bien no a la manera de medios externos, sino como su expresión real más genuina:

La idea es así, en primer lugar, la vida, el concepto que, por diferir de su objetividad y, como fin de sí mismo, tiene en ella su propio medio y la pone como su propio medio, pero estando dentro de este medio de manera inmanente, y siendo allí el fin realizado [realisierte], idéntico consigo ${ }^{52}$.

La Idea es el fin que se realiza en el objeto y deja de tener, por tanto, un $o b$-jeto en el sentido de algo contrapuesto; ni la célula, ni el órgano, ni el organismo en su conjunto pueden ser considerados como meros "objetos" de la Vida, sino como su propia realización objetiva. En tanto que realización de la Vida, el organismo es el "medio" perfectamente adecuado al fin o el fin que coincide plenamente con su "objeto" 53 . Pues bien: esta unidad de lo subjetivo y

${ }^{51}$ Id., 172: "Diese Identität ist einerseits der einfache Begriff und [die] ebenso unmittelbare Objektivität, aber andererseits gleich wesentlich Vermittlung und nur durch sie als sich selbst aufhebende Vermittlung jene einfache Unmittelbarkeit; so ist er wesentlich dies, als fürsichseiende Identität von seiner ansichseien-den Objektivität unterschieden zu sein und dadurch Äußerlichkeit zu haben, aber in dieser äußerlichen Totalität die selbstbestimmende Identität derselben zu sein. So ist der Begriff nun die Idee."

52 Ibid., 177 / II, 477 (Mondolfo) / II, 316 (Duque): "So ist die Idee erstlich das Leben; der Begriff, der unterschieden von seiner Objektivität einfach in sich seine Objektivität durchdringt und als Selbstzweck an ihr sein Mittel hat und sie als sein Mittel setzt, aber in diesem Mittel immanent und darin der realisierte mit sich identische Zweck ist."

${ }^{53}$ Ibid., 184: "Da ihm der Begriff immanent ist, so ist die Zweckmäßigkeit des Lebendigen als innere zu fassen; er ist in ihm als bestimmter, von seiner Äußerlichkeit unterschiedener und in seinem Unterscheiden sie durchdringender und mit sich identischer Begriff. Diese Objektivität des Lebendigen ist Organismus; sie ist das Mittel und Werkzeug des Zwecks, vollkommen zweckmäßig, da der Begriff ihre Substanz ausmacht; aber eben deswegen ist dies Mittel und Werkzeug selbst der ausgeführte Zweck, in welchem der subjektive Zweck insofern unmittelbar mit sich selbst zusammengeschlossen ist." 
lo objetivo es, a mi juicio, lo que expresa Hegel mediante la expresión híbrida "realisierte Zweck", en la que el lado objetivo se enfatiza mucho más que en la expresión "erfüllte Zweck".

No querría concluir esta nota sin hacer una breve reflexión sobre el significado que esta reedición de la Ciencia de la Lógica tiene en una época como la nuestra, que se autoconcibe como esencialmente postmetafísica y que, por lo general, sólo se interesa por Hegel a condición de renunciar al trasfondo metafísico de su filosofía. ¿Cómo interpretar la reaparición de esta obra (cuya parte esencial Hegel identifica con la "metafísica propiamente dicha" (eigentliche Metaphysik $)^{54}$ ) en una época que no distingue entre metafísica y dogmatismo, y a cuyos ojos no aparece más que como un edificio mortuorio? ¿Se trata de una reaparición puramente conmemorativa, como fácilmente podría colegirse del hecho de que su publicación coincida prácticamente con la efeméride de su centenario? ¿O responde más bien a un interés doxográfico, propiciado por los avances de la Gesammelte Werke? Ya hemos aludido a la oportunidad que la monumental edición crítica ofrece a este tipo de publicaciones, pero todavía queda por explicar el impulso que alienta a una época como la nuestra a coger el tren de esa oportunidad, emprendiendo el titánico esfuerzo de reunir y traducir los materiales dispersos en torno a ella.

Parece claro que un esfuerzo como éste no puede provenir de un interés puramente conmemorativo o doxográfico: más bien parece como si respondiera a la necesidad de saldar cuentas con los tópicos del "cierre" y la "clausura" que vertebran la lectura más generalizada en el ámbito del hegelianismo postmetafísico. Se percibe en él, por tanto, algo del primer impulso que alentó el denominado "Hegel-renaissance" en los años 30 del pasado siglo, y que fue suscitado por el descubrimiento de los escritos de juventud. Como es sabido, éstos mostraban a un Hegel diferente de aquella escolástica hegeliana tan fácilmente asimilable a los parámetros de la identidad y de la onto-teología, si bien sólo al precio de trazar una artificiosa distinción entre un primer y un segundo Hegel.

Una distinción como ésta es, sin embargo, lo que la Gesammelte Werke parece difuminar ahora, al descubrir la misma viveza y dinamismo característicos de los primeros escritos en la obra más sistemática y madura de Hegel. Como no podía ser de otra manera, ello propicia una nueva recepción de la misma, libre de los mencionados prejuicios de la identidad y de la clausura, más achacables a la Sämmtliche Werke de los discípulos que a Hegel mismo.

${ }^{54} G W 11,7$. 
Corresponde a las próximas décadas decidir el signo de esta nueva recepción que, a día de hoy, se encuentra en una encrucijada semejante a la que se perfilaba en los años treinta del pasado siglo, cuando las lecturas francesas de la Fenomenología dejaban entrever a un Hegel conmensurable en muchos puntos con los parámetros de la hermenéutica y el pensamiento postmetafísico, pero también, por la misma razón, a un Hegel inconmensurable e inatacable desde esas mismas coordenadas.

Como si de un señuelo se tratara, este nuevo Hegel atrae a sus críticos al vórtice de su dialéctica para devorarlos y asimilarlos en el metabolismo de su Sistema. La razón de que ello haya permanecido prácticamente inexplorado por el hegelianismo del siglo xx es algo que no puede dirimirse en esta nota. Nos conformaremos con constatar la nueva oportunidad que sus epígonos encuentran hoy en la edición de la $G W$, que ofrece la posibilidad de comprender más detalladamente el proceso de gestación de la obra más sistemática de Hegel, de la que hasta ahora sólo conocíamos el resultado cerrado y acabado con el que su autor quiso legarla. Como no podía ser de otra manera, ello supone (sobre todo) una nueva oportunidad para la Ciencia de la lógica, lastrada hasta ahora por una lectura cargada de prejuicios que impedían ver las implicaciones, tanto de la "exposición crítica" de la metafísica dogmática que Hegel despliega en la "Lógica objetiva", como de la nueva metafísica que ésta hace posible y ve la luz en la "Lógica subjetiva" 55 .

Considero que lo que ahí recibe el nombre de "metafísica propiamente dicha" permanece todavía incomprendido por el hegelianismo postmetafísico que, al igual que los críticos de Hegel, asume por lo general la misma identificación de "metafísica" y "dogmatismo", y que es precisamente el interés por explorar esta nueva configuración de la metafísica lo que alienta el interés por la Ciencia de la lógica en nuestros días. Me atrevo a pronosticar que, así como el hegelianismo del siglo xx gravitó en torno al estudio de la Fenomenología, el del siglo Xxi lo hará en torno esta gran obra, injustamente considerada como una suerte de "mausoleo" o "edificio mortuorio", y que el resultado de este estudio no se limitará únicamente al descubrimiento de algunos detalles más o menos conmensurables con los parámetros del pensamiento postmetafísico, sino que se saldará con su asunción y consiguiente superación.

${ }^{55}$ Cf. supr. nota 54. 


\section{Bibliografía}

Hegel, G. W. F. Gesammelte Werke [GW] (in 21 Bände), in Verbindung mit der Deutschen Forschungsgemeinschaft hrsg. von der Rheinisch-Westfälischen Akademie der Wissenschaften und der Künste, Hamburg: Felix Meiner, desde I968.

—GW 11: Wissenschaft der Logik. Erster Band. Die objektive Logik (I 8 I 2, I3). Hrsg. Von F. Hogemann y W. Jaeschke, I978.

—GW 12: Wissenschaft der Logik. Zweiter Band. Die subjektive Logik (I8 I6). Hrsg. Von F. Hogemann y W. Jaeschke, I98 I.

—GW 21: Wissenschaft der Logik. Erster Band. Die objektive Logik (I 832). Hrsg. Von F. Hogemann y W. Jaeschke, I978.

\section{Traducciones cotejadas}

Castellanas:

Mondolfo, R. (trad.) 1956, Ciencia de la lógica, Vols. I-II, Buenos Aires: Hachette.

DuQue, F. (trad.) 20 i I, I 5, Ciencia de la lógica, Vols. I-II, Madrid: Abada.

Francesas:

Jankélévitch, V. (trad.) i947, Science de la logique, Vols. I-II, París: Aubier.

Labarrière, P.-J. y JarczyK, G. (trad.) i972, 8 I, Science de la logique, Vols. I-II, París: Aubier.

Inglesas:

Johnston, W. H. y Struthers, L. G. (trad.) i929, Science of logic, Vols. I-II, Nueva York: Humanities Press.

Giovanni, G. Di (trad.) 20io: Science of logic, Cambridge: Cambridge University Press.

Italianas:

Moni, A. (trad.) I 924, 25, 25, Scienza della logica, Vols. I-III, Bari: Laterza \& Figli.

Giuspoli, P., Castegnaro, G. y Livieri, P. (trad.) 2009, La scienza della logica, Vols. I-III, Trento: Verifiche. 
Otras referencias:

DuQue, F. 1985-87, I990, La especulación de la indigencia, Barcelona: Juan Granica.

Duque, F. 1998, Historia de la filosofía moderna. La era de la critica, Madrid: Akal. Fabo Lanuza, J. 2007, Reflexión o diferencia. Un estudio sobre la cuestión Hegel y el problema de la metafísica, tesis doctoral inédita, Universidad Complutense de Madrid. 